\title{
A case of double alleles at three Y-STR loci: forensic implications
}

Received: 27 September 2004 / Accepted: 3 January 2005 / Published online: 3 February 2005

(C) Springer-Verlag 2005

\begin{abstract}
In a genetic study of unrelated donors from Bahia (Brazil), one sample contained a 16 Y-STR haplotype with double peaks at three loci: DYS389 II, DYS437 and DYS439. The son of the subject had the same haplotype as found in the father. This profile was compared with a similar case found in a paternity case investigation in Madrid (Spain) and a match was found for the full 16 Y-STR haplotype. Because these three loci are located within the AZFa segment, these results are in accordance with duplication of the AZFa region that includes also other Y-STRs currently used in forensic investigation, for example DYS389I and DYS438. This case attracts our attention in the forensic interpretation of Y-haplotype profiles, because multiple alleles at various loci do not indicate forcibly that the sample under analysis is a mixture.
\end{abstract}

Keywords Duplication · Y-STR · AZFa region · Human identification

M. Diederiche $\cdot$ F. Corte-Real

Serviço de Biologia Forense,

Instituto Nacional de Medicina Legal,

Coimbra, Portugal

P. Martín

Instituto Nacional de Toxicología y Ciencias Forenses,

$\mathrm{M}^{\circ}$ de Justicia,

Madrid, Spain

A. Amorim · L. Gusmão $(\bowtie)$

IPATIMUP,

Rua Dr. Roberto Frias, s/n,

4200-465 Oporto, Portugal

e-mail: lgusmao@ipatimup.pt

Tel: $+351-22-5570700$

Fax: +351-22-5570799

A. Amorim

Faculdade de Ciências, Universidade do Porto,

Oporto, Portugal

F. Corte-Real

Faculdade de Medicina, Universidade de Coimbra,

Coimbra, Portugal

\section{Introduction}

In the forensic field, Y-STRs have been shown to be particularly helpful for detecting a male DNA fraction in stains involving male/female mixtures, enabling direct determination of the Y-haplotype without the need for differential extraction. In multiple rape cases Y-STRs are often used for determination of the number of contributors. The presence of additional alleles in a $\mathrm{Y}$ chromosome profile is usually interpreted as a mixture of more than one contributor. However, concerning the most used Y-STRs in forensics, there are already many reports in which two alleles were found for a single locus, namely in DYS19, DYS389, DYS390, DYS391, DYS393 and DYS385 (e.g. Kayser et al. 1997, 2000; Kurihara et al. 2004; Çakir et al. 2004; YHRD, http://www.yhrd.com). In all these examples, except for DYS385, in a single male haplotype only one locus showed duplicated alleles. Bosch and Jobling (2003) described three cases of YSTR multiple alleles due to duplication of the AZFa region followed by mutations in DYS389II and/or DYS439.

Precise estimation of the frequency of duplicatedmutated YSTR alleles is very important in forensic genetic analysis, because the presence of multiple peaks can be misinterpreted as mixed profiles.

Here, we describe a case in which a father-son pair presented double peaks in the DYS389 II, DYS437 and DYS439 loci, simultaneously, after analysis of 16 Y-STR.

\section{Case report}

In a population genetic study of 72 unrelated donors from Bahia (Brazil), one sample showed a rare Y chromosome STR profile with three loci presenting double peaks. To exclude the possible presence of a mixed profile because of sample contamination, a second sample was collected and analysed giving an identical result. The same Y-STR haplotype was also obtained after analysis of a sample from his son. These two individuals (father and son) are apparently healthy and no cases of infertility are known in the 
family. A karyotype analysis showed the presence of a single Y chromosome.

The present profile was compared with a similar case found during a paternity case investigation in Madrid (Spain) and a match was found for the full 16 Y-STR haplotype.

\section{Materials and methods}

DNA was extracted from bloodstains by Chelex or standard phenol/chloroform procedures and quantified by slot-blot hybridization using the Quantiblot Human DNA Quantitation kit (AB, Applied Biosystems). Each sample was amplified using two different PCR amplification protocols, to confirm the DYS389, DYS437 and DYS439 results with different primer pairs. PCR reactions were performed according to the protocols described by Kayser et al. (1997), Schneider et al. (1998), Gusmão et al. (1999) and SanchezDiz et al. (2003), or by using Y-Plex 6 and Y-Plex 5 (Reliagene) and PowerPlex Y System (Promega Corporation) kits. Detection was carried out on an ABI Prism 310 Genetic Analyzer instrument (AB, Applied Biosystems). Alleles separation and the sequencing reaction were according to Gusmão et al. (2002).

\section{Results and discussion}

In a male sample from Bahia (Brazil) we observed, out of the 16 Y-STRs typed, double peaks in the DYS437, DYS439 and DYS389 II alleles' range. The same 16 YSTR haplotype was also found in his son and in a male sample from Spain (Fig. 1).

Sequence analysis confirmed the number of repeats attributed (Table 1).

The most likely explanation of the observation of this haplotype is the presence of segment duplication involving, at least, these three loci. This duplication should have occurred many generations ago, enough for accumulation of
Table 1 DYS389 II, DYS437 and DYS439 alleles' structure observed in the three studied samples, carrying the following 16 Y-STR haplotype: DYS19 (14), DYS385 (12-13), DYS389 I (13), DYS389 II (29-30), DYS390 (23), DYS391 (11), DYS392 (13), DYS393 (13), DYS437 (14-15), DYS438 (12), DYS439 (10-11), DYS460 (10), DYS461 (13), GATA A10 (14), GATA C4 (23) and GATA H4 (28)

\begin{tabular}{lll}
\hline Marker & Allele & Repetitive sequence structure \\
\hline DYS389 II & 29 & $\ldots(\text { TCTG })_{5}(\text { TCTA })_{11} \ldots(\text { TCTG })_{3}(\text { TCTA })_{10} \ldots$ \\
& 30 & $\ldots(\text { TCTG })_{5}(\text { TCTA })_{12} \ldots(\text { TCTG })_{3}(\text { TCTA })_{10} \ldots$ \\
DYS437 & 14 & $\ldots(\text { TCTA })_{8}(\text { TCTG })_{2}(\text { TCTA })_{4} \ldots$ \\
& 15 & $\ldots(\text { TCTA })_{9}(\text { TCTG })_{2}(\text { TCTA })_{4} \ldots$ \\
DYS439 & 10 & $\ldots(\text { GATA })_{10} \ldots$ \\
& 11 & $\ldots(\text { GATA })_{11} \ldots$ \\
\end{tabular}

the three STR mutations detectable by the presence of double peaks. These three loci are, indeed, located in the same Y-chromosome region, the $780 \mathrm{~kb}$ AZFa segment (Bosch and Jobling 2003), together with other Y-STRs, two of them also typed in the samples in the current work (DYS389 I and DYS438).

Bosch and Jobling (2003) described the AZFa duplications as the reciprocal product of recombination between HERVs flanking the region and they estimate a frequency of $0.17 \%$ in Iberia. By Y-STR analysis these authors reported three chromosomes carrying the duplication, by the detection of double peaks in DYS389 II and DYS439. In the current case, double peaks were observed in the same markers, and in DYS437. The subsequent accumulation of three mutation events after duplication suggests that it has persisted for several generations. When tracing the paternal ancestry of the Brazilian subject it was found that his grandfather came from Galicia in Spain. Therefore, given the exact match observed between the Brazilian and Spanish samples, it is likely that the ancestral duplication event occurred in Iberia.

Y-STR information in personal identification has been recognized as a powerful tool for detecting the male profile in mixtures with a high proportion of female DNA. These

Fig. 1 Y-STR haplotype profile showing the presence of additional alleles at DYS389 II, DYS437, and DYS439, after amplification with the PowerPlexY kit

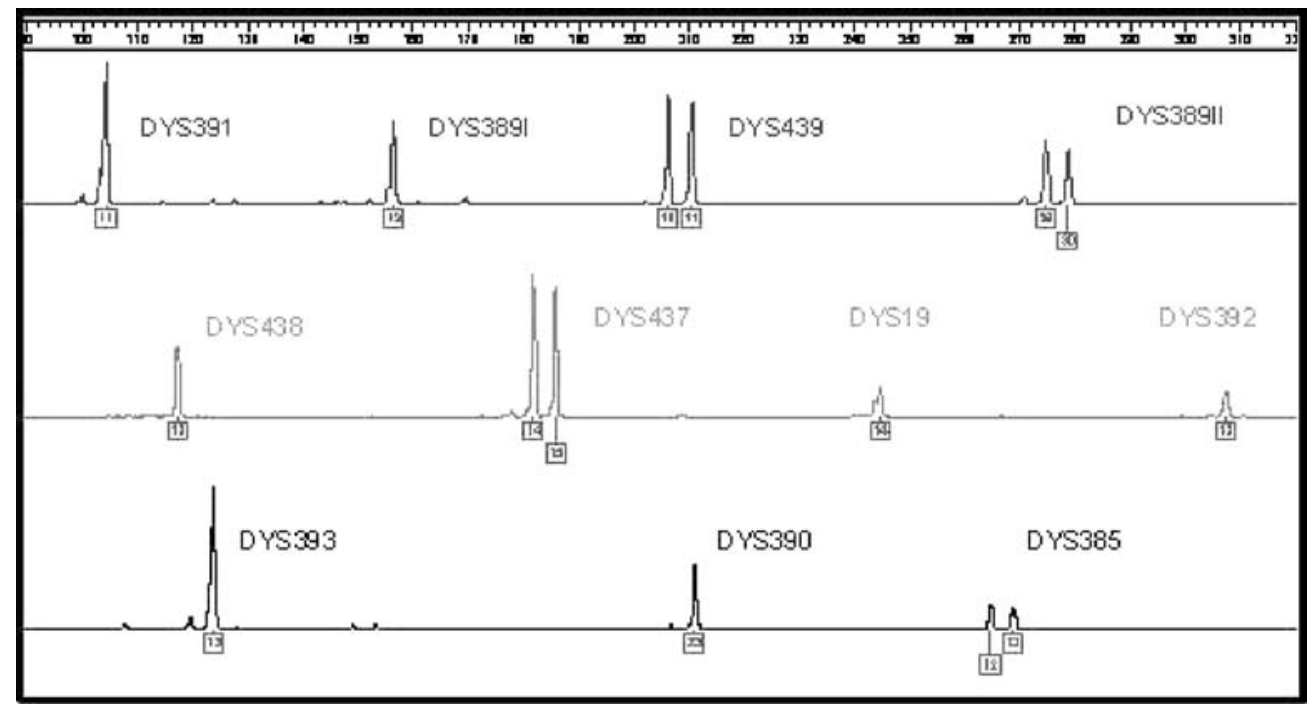


mixtures are very common in rape cases where the $\mathrm{Y}$ profile is also used to infer the number of male contributors. The most used Y-STRs in forensic analysis are DYS19, DYS389 I and II, DYS390, DYS391, DYS392, DYS393 and DYS385. For most of these, examples were reported of double peaks for a single locus because of a duplication event followed by a mutation. Therefore, the presence of double alleles does not always mean the presence of multiple male profiles, and so it is important to know the incidence of duplication-mutation involving the Y-STRs in forensic investigations.

Considering the STRs located in the AZFa region, only DYS389 is included in the minimum haplotype (YHRD). However, other markers located in this segment are in the set selected by The Scientific Working Group on DNA Analysis Methods (SWGDAM) for forensic DNA analysis in the US, and in commercial kits. Therefore, although there have been few reports of the presence of double alleles in more than one locus in the same chromosome, because of the frequency of duplication of the AZFa region (Bosch and Jobling 2003) it is expected that, with the simultaneous amplification of Y-STRs located in this region, other cases will be soon described.

Acknowledgements Fundação para a Ciência e a Tecnologia, Programa Operacional Ciência, Tecnologia e Inovação (POCTI); CNPq/Brazil, Universidade Estadual de Santa Cruz and Departamento de Polícia Técnica-SSP/BA. Special thanks to the donors of the samples.

\section{References}

Bosch E, Jobling MA (2003) Duplications of the $A Z F a$ region of the human Y chromosome are mediated by homologous recombination between HERVs and are compatible with male fertility. Hum Mol Genet 12(3):341-347
Çakir AH, Celebioglu A, Yardimci E (2004) Y-STR haplotypes in Central Anatolia region of Turkey. Forensic Sci Int 144(1):5964

Gusmão L, Gonzalez-Neira A, Pestoni C, Brion M, Lareu MV, Carracedo A (1999) Robustness of the Y STRs DYS19, DYS389 I and II, DYS390 and DYS393: optimization of a PCR pentaplex. Forensic Sci Int 106(3):163-172

Gusmão L, González-Neira A, Alves C, Lareu M, Costa S, Amorim A, Carracedo A (2002) Chimpanzee homologous of human Y specific STRs. A comparative study and a proposal for nomenclature. Forensic Sci Int 126(2):129-136

Kayser M, Caglia A, Corach D, Fretwell N, Gehrig C, Graziosi G, Heidorn F, Herrmann S, Herzog B, Hidding M et al (1997) Evaluation of Y-chromosomal STRs: a multicenter study. Int J Leg Med 110:125-133, 141-149

Kayser M, Roewer L, Hedman M, Henke L, Henke J, Brauer S, Krüger C, Krawczak M, Nagy M, Dobosz T et al (2000) Characteristics and frequency of germline mutations at microsatellite loci from the human Y chromosome, as revealed by direct observation in father/son pairs. Am J Hum Genet 66: $1580-1588$

Kurihara R, Yamamoto T, Uchihi R, Li SL, Yoshimoto T, Ohtaki H, Kamiyama K, Katsumata Y (2004) Mutations in 14 Y-STR loci among Japanese father-son haplotypes. Int J Leg Med 118: 125-131

Sanchez-Diz P, Gusmão L, Beleza S, Benitez-Paez A, Castro A, Garcia O, Solla LP, Geada H, Martin P, Martinez-Jarreta B et al (2003) Results of the GEP-ISFG collaborative study on two Y-STRs tetraplexes: GEPY I (DYS461, GATA C4, DYS437 and DYS438) and GEPY II (DYS460, GATA A10, GATA H4 and DYS439). Forensic Sci Int 135:158-162

Schneider PM, Meuser S, Waiyawuth W, Seo Y, Rittner CV (1998) Tandem repeat structure of the duplicated Y-chromosomal STR locus DYS385 and frequency studies in the German and three Asian populations. Forensic Sci Int 97:61-70 